\title{
Vision-Based Displacement Sensor for Monitoring Dynamic Response Using Robust Object Search Algorithm
}

\author{
Yoshio Fukuda, Maria Q. Feng, Yuto Narita, Shun'ichi Kaneko, and Takayuki Tanaka
}

\begin{abstract}
This paper develops a vision-based displacement measurement system for remote monitoring of vibration of largesize structures such as bridges and buildings. The system consists of one or multiple video cameras and a notebook computer. With a telescopic lens, the camera placed at a stationary point away from a structure captures images of an object on the structure. The structural displacement is computed in real time through processing the captured images. A robust object search algorithm developed in this paper enables accurate measurement of the displacement by tracking existing features on the structure without requiring a conventional target panel to be installed on the structure. A sub-pixel technique is also proposed to further reduce measurement errors cost-effectively. The efficacy of the vision system in remote measurement of dynamic displacements was demonstrated through a shaking table test and a field experiment on a long-span bridge.
\end{abstract}

Index Terms - Image processing, remote monitoring, structure health monitoring, template matching.

\section{INTRODUCTION}

C IVIL engineering structures including bridges and buildings are exposed to various external loads such as traffic, earthquakes, and winds during their lifetime, and monitoring structural response displacements to such dynamic loads plays an important role in assessing the on-going structural integrity and preventing catastrophic failure. Conventionally, contacttype sensors such as linear variable differential transformers (LVDT's) or dial gauges are employed for measuring structural displacements, but they need to be installed between the measurement point of the structure and a stationary platform as a reference point, which is difficult, and sometimes impossible for a large-scale civil engineering structure, such as a bridge spanning a wide river or a deep canyon. Moreover, these sensors usually need to be wired to external devices such as a data logger and a power-supply unit. Such cabling is cumbersome and costly, particularly for monitoring a large structure. On the other hand, accelerometers, which do not require a stationary

Manuscript received November 14, 2012; revised March 1, 2013 and May 31, 2013; accepted July 3, 2013. Date of publication July 15, 2013 date of current version October 4, 2013. The associate editor coordinating the review of this paper and approving it for publication was Dr. Anna Grazia Mignani.

Y. Fukuda and M. Feng are with the Department of Civil Engineering and Engineering Mechanics, Columbia University, New York, NY 10027 USA (e-mail: yf2290@columbia.edu; mfeng@columbia.edu).

Y. Narita, S. Kaneko, and T. Tanaka are with the Division of Systems Science and Informatics, Graduate School of Information Science and Technology, Hokkaido University, Hokkaido 153-8505, Japan (e-mail: narita@ssc.ssi.ist.hokudai.ac.jp; kaneko@ssi.ist.hokudai.ac.jp; ttanaka@ssi.ist.hokudai.ac.jp).

Digital Object Identifier 10.1109/JSEN.2013.2273309 reference point, can be used to measure displacements through double integration of acceleration [1], [2], but numerical errors are of concern, and sensor installation and cabling are still probmatic. The Laser Doppler vibrometers and global positioning systems (GPS) have potential advantage for the application in this field, but the total cost of the system tends to be expensive [3]-[8].

To overcome these problems, displacement sensors based on video image processing, the so-called vision-based displacement sensros, have recently been developed [9]-[12]. These sensors, including the low-cost system previously developed by the authors [12] require an installation of a target maker panel on the measurement point of a structure. The maker with a pre-designed high-contrast black and white pattern enables a high-precision tracking of the movement of the target panel by a video camera, and thus an accurate measurement of the structural displacement time histories. The use of a commercial digital camcorder and a personal computer reduces the total cost of the whole system. It is noted that such a system measures the relative displacement between the target panel and the ground support of the camera. Whereas the non-contact, remote displacement measurement represents significant advance over the conventional contact sensors, the installation of the target panel still requires access to the structure and the panel needs weather protection for longterm monitoring. Most recently, several systems without the targets have been developed, but their measureable locations on the structure are limited to specific characteristics such as cables [13], [14] and gusset plates [15], and some suffer from accuracy problems compared with conventional sensors [16]. Moreover, measurement of structural dynamic responses with the vision-based systems has limitations in the maximum sampling rate and measurement accuracy, due primarily to the camera capability.

The objective of this study is to eliminate the target panel and instead to use existing features such as edges and rivets on a structure to achieve accurate measurement of structural displacements under dynamic loads. This represents a significant technical challenge. The lack of contrast and changes in shading, lighting, and background conditions in the field make it highly difficult to accurately extract displacements from video images using the conventional template matching techniques, such as the correlation coefficient and the sum of squared difference. Furthermore, the template matching algorithm must be computationally efficient for real-time meaurement. Finally, techniques are desired to maximize the camera capability 


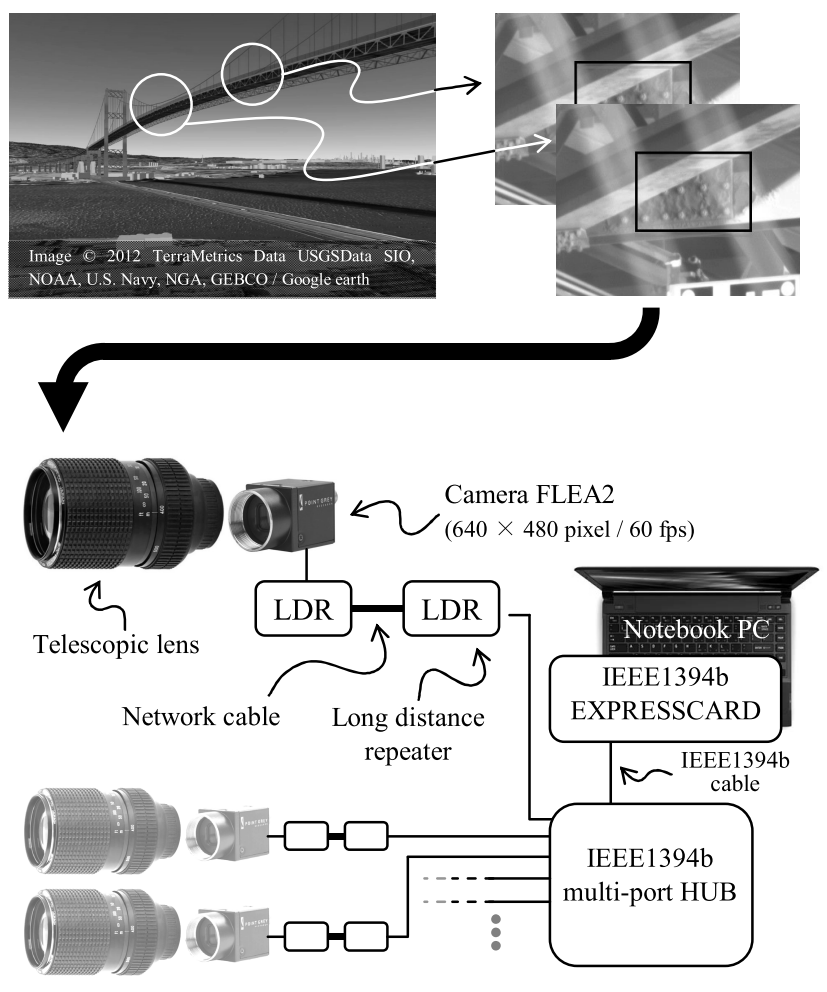

Fig. 1. Vision-based remote displacement sensor.

(such as the resolution) employed in the measurement system. In this study, we developed and applied a novel orientation code matching technique, an object search algorithm robust to the changes in the object surrounding conditions. Structural displacements thus measured at a long-span bridge were compared with those by tracking a target panel. The accuracy of the measured displacement was validated by a comparison with LVDT in a seismic shaking table test.

\section{SEnSOR System}

The vision-based sensor system developed in this study, as shown in Figure 1, consists of a notebook computer connected to one or multiple video cameras with telescopic lenses, which can measure structural displacements at different locations simultaneously. The video images of a target on a structure, such rivets and edges, captured by the camera are digitized into $640 \times 480$ pixel images in 8 bit grey scales and streamed into the notebook computer through an IEEE connection and a network cable via a long distance repeater with a maximum sampling rate of 60 frames per second. The network cable and the long distance repeater enable an extension of the distance between the camera and the computer up to $100 \mathrm{~m}$. This capability improves the flexibility of the installation in the actual field measurement. Moreover, by using an IEEE 1394b multi-port hub, more than one cameras can be connected to one computer and controlled at same time. By processing the digital video images on the computer, a time history of the displacement of the target on the structure is obtained.

For accurate measurement of the dynamic displacement in field conditions, the Orientation Code Matching (OCM) algorithm was further developed and applied for video image processing. The algorithm is based on matching gradient information, around each pixels computed in the form of orientation codes, rather than the gray levels directly. Application of gradient information has been previously studied for finding texture orientation [17] and recognizing gesture [18]. A recent study by the project team has shown that the matching can be made robust by using only difference or tendency of adjacent pixel brightness values rather than their actual values [19], based on which the OCM algorithm was proposed and formulated [20]-[23].

\section{ORientation Code Matching}

In the OCM scheme, orientation code representations for an object image and the template are constructed from the corresponding gray images such that each pixel represents an orientation code that is obtained by quantizing the orientation angle at the corresponding pixel position in the gray image. The orientation angle represents the steepest ascent orientation evaluated from the pixel neighborhoods, and measured with respect to the horizontal axis. The orientation codes thus obtained are a function of the texture and shape of the object and hence essentially invariant to object translation and the effects of shading, background and illumination variations. The detail of the OCM technique with a pixel level analysis is shown below.

Suppose an analog image is represented by $I(x, y)$ and its horizontal and vertical derivatives as $\nabla I_{x}=\frac{\partial I}{\partial x}$ and $\nabla I_{y}=\frac{\partial I}{\partial y}$, respectively. For the discrete version of the image, they are evaluated around a pixel position $(i, j)$, then orientation angle $\theta_{i, j}$ is computed by using the $\tan ^{-1}$ function as $\theta_{i, j}=\tan ^{-1}\left(\nabla I_{y} / \nabla I_{x}\right)$. Since the numerical value of $\tan ^{-1}$ function is confined to $\left[\frac{-\pi}{2}, \frac{\pi}{2}\right]$, the actual orientation is determined after checking signs of the derivatives, thus making the range of $\theta$ to be $[0,2 \pi]$.

The orientation code is obtained by quantizing $\theta_{i, j}$ into $N\left(=2 \pi / \Delta_{\theta}\right)$ levels with a constant width $\Delta_{\theta}$. We have to choose an appropriate value of the width for precise detection of template instances in the scene. This issue should be considered in relation to inherent information amount and possible spatial resolution.

The code is defined as below using the Gaussian notation:

$$
c_{i, j}= \begin{cases}{\left[\frac{\theta_{i, j}}{\Delta \theta}\right]:} & \left|\nabla I_{x}\right|+\left|\nabla I_{y}\right|>\Gamma \\ N=\frac{2 \pi}{\Delta_{\theta}}: & \text { otherwise }\end{cases}
$$

where $\Gamma$ is a pre-specified threshold level for ignoring the low contrast pixels and $\Gamma$ is a large value, which is assigned as a code for them. The purpose of using $\Gamma$ is to prevent the uniform or semi-uniform regions from influencing the error evaluation as the pixels with low contrast neighborhoods are more sensitive to noise. Using a too large value of $\Gamma$ can cause the suppression of information in low contrast images (containing strongly shaded or illuminated objects). An example of the orientation codes is depicted in Figure 2 corresponding to the width for quantization of $\Delta_{\theta}=\pi / 8$. When the contrast is lower than the threshold $\Gamma$, then $N=\frac{2 \pi}{\Delta_{\theta}}$ as invalid code is assigned to the orientation code $c_{i, j}$.

A dissimilarity measured based on the definition of orientation codes is designed to evaluate the deference between 


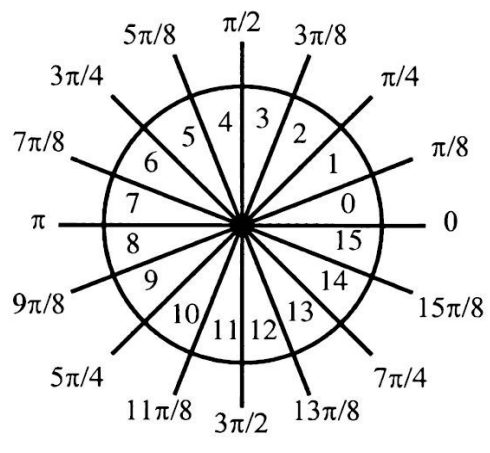

Fig. 2. Orientation codes.

any two images of the same size. The best match between orientation code images of the template $T$ and any object image $I$ from the same scene is searched by minimizing the measured in the form of summation of error function as follows:

$$
D=\frac{1}{M} \sum_{I_{m, n}} d\left(O_{I_{m, n}}(i, j), O_{T}(i, j)\right)
$$

where $O_{I_{m, n}}$ and $O_{T}$ are the orientation code images of the sub image and the template respectively, $M$ is the size of the template, $(m, n)$ shows the position of the sub image in the scene, and $d(\cdot)$ is the error function based on an absolute difference criterion

$$
d(a, b)=\left\{\begin{aligned}
\min \{|a-b|, N-|a-b|\} & \\
& \text { if } a \neq N \cap b \neq N \\
\frac{N}{4}, & \text { if } a \neq N \cap b=N \\
0, & \text { otherwise. }
\end{aligned}\right.
$$

When a comparison is performed between a pixel having an orientation code evaluation by the $\tan ^{-1}$ function and the one whose code was set to $N$ due to low contrast neighborhood, the error cannot be computed by finding the difference. In order to avoid such an inconsistent comparison, we need to assign a reasonable value to the error function corresponding to such pixels. The assigned value should be such that it does not bias the dissimilarity evaluation for the sub image. For such cases, we assigned the value of $\frac{N}{4}$ to error function. This is the error value which is expected when these two pixels have no relation between each other. A large value for $N$ is helpful for discriminating such an incompatible comparison.

Since the orientation code are cyclic in nature, the absolute difference is not used directly for computing the error function, rather the minimum distance between the two codes is determined. For example, in Figure 2, the code 0 is only a unit difference away from the code 15 , whereas their simple difference yields 15. Such evaluation makes the matching stable against minor pose variations of the object, regardless of the direction of the movement. As a consequence of this cyclic property of orientation codes, the maximum distance between any two codes is never more than $\frac{N}{2}$.

Finally, the similarity ratio $s$ is derived as follows:

$$
\begin{aligned}
& h=\frac{D}{\frac{N}{2}} \quad(0 \leq h \leq 1) \\
& s=1-h \quad(0 \leq s \leq 1)
\end{aligned}
$$

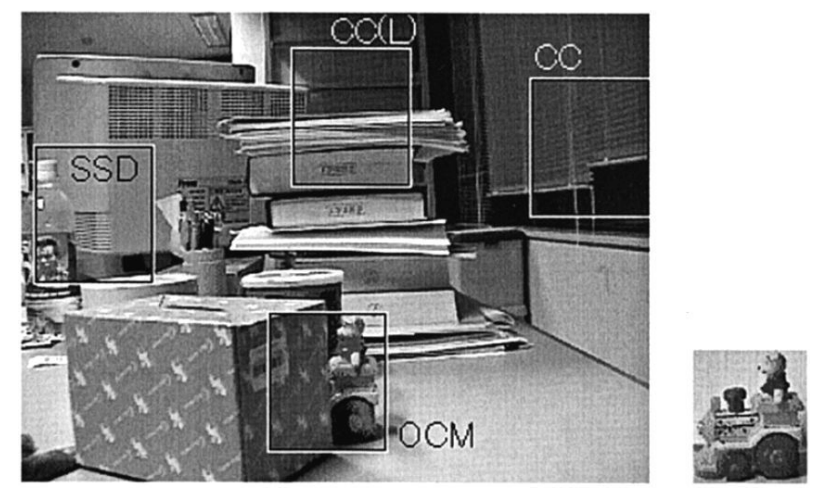

Fig. 3. Comparison of OCM with CC \& SSD.

where $h$ is discrimination ratio obtained by dividing the average absolute difference $D$ by the maximum of absolute difference $\frac{N}{2} . s$ is the similarity ratio. To find the best matched point, at which the similarity reaches the maximum value, the template image must be compared with the entire target frame pixel by pixel. This is obviously time consuming. In order to drastically reduce the computational time, a ROI window is defined based on the currently best-matched point, and the OCM processing is carried out only within this limited window.

To evaluate the effectiveness of the OCM technique in dealing with shading and background shift, a toy bear was selected as the object of interest and test images were prepared involving various conditions of shading, background, occlusion and their combinations. As shown in Figure 3, the OCM technique successfully detected the correct position of the target toy, which is partially occluded and shaded, whereas conventional template matching techniques such as the correlation coefficient (CC) and sum of squared difference (SSD) techniques failed to do so. The $\mathrm{CC}$ technique evaluated in this test is generally used for the digital image correlation (DIC) technique, which is widely applied for the measurement of the image deformation and displacement. It is expected that the integration of the OCM and the DIC techniques would improves the performance of DIC by eliminating its surface pattern requirement, and enable not only displacement measurement but also deformation analysis from a high-resolution image that covers a larger area of the target structure.

The processing time of the OCM technique tends to be longer than the conventional template matching technique. However, by estimating the maximum movement range of the target location between two frames (16 ms for image capturing with $60 \mathrm{fps}$ ), it is possible to re-configure a region of interest (ROI) in next frame. The OCM processing is applied only in this ROI. During the measurement, by properly updating this ROI in each frame based on the current detected target location, the amount of processing time is dramatically reduced.

\section{Shaking Table Test}

In order to evaluate the performance of the OCM technique, including the pixel level accuracy and the target trackability of a dynamic response, a laboratory test was carried out using 

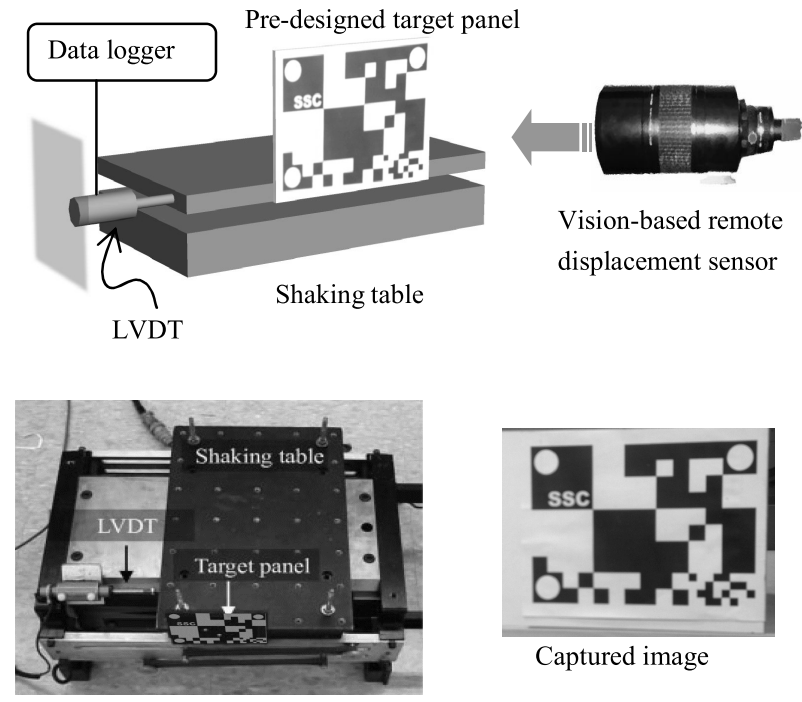

Electromagnetic shaking table

Fig. 4. Setup of shaking table test.

a seismic shaking table subjected to sinusoidal motion with different frequencies and seismic ground motion. The visionbased remote displacement sensor system was experimentally evaluated in comparison with a conventional LVDT, a contact sensor, during the shaking table test. Figure 4 shows the test setup, in which a pre-designed black and white target panel $(216 \mathrm{~mm} \times 280 \mathrm{~mm})$ was fixed to an electromagnetic shaking table, and the LVDT was installed between the shaking table and a stationary reference point. A video camera with a $75 \mathrm{~mm}$ lens was placed at a stationary position 5.5 meters away from the shaking table. The camera captures the target panel with 60 frames per second and detects the displacement of the target panel by performing the OCM technique with a pixel level analysis on a connected computer. Before the measurement, the actual pixel size of a digital image was measured by using the pre-designed target panel. The size of a pixel was calibrated to $0.54 \mathrm{~mm} / \mathrm{pixel}$ and the theoretical value of the error by the measurement is $0.27 \mathrm{~mm}$.

The shaking table was first driven by sinusoidal signals of different frequencies ranging from $0.1 \mathrm{~Hz}$ to $5.0 \mathrm{~Hz}$, with a constant amplitude of $50.0 \mathrm{~mm}$. At each frequency, measurement was taken for 60 seconds. The displacement time histories obtained by processing the video images captured by the camera were compared with those measured by the LVDT. An excellent agreement was observed at all the tested frequencies. Figure 5 shows examples at $1.0 \mathrm{~Hz}$, $2.5 \mathrm{~Hz}$, and $5.0 \mathrm{~Hz}$. Furthermore, the shaking table was driven by a recorded earthquake ground motion, the 1995 Great Hanshin-Awaji earthquake, and the table displacement time history measured by the vision-based sensor agrees well with that by the LVDT, as shown in Figure 5.

To evaluate the measurement error in the vision-based sensor system, the standard deviation between the displacements measured by these two sensor systems is plotted in Figure 8 for each of the sinusoidal frequencies. The maximum error is $0.21 \mathrm{~mm}$, which is sufficiently small considering the motion amplitude of $50.0 \mathrm{~mm}$. And the maximum error nearly equals to the theoretical value based on a pixel level analysis, thus it is

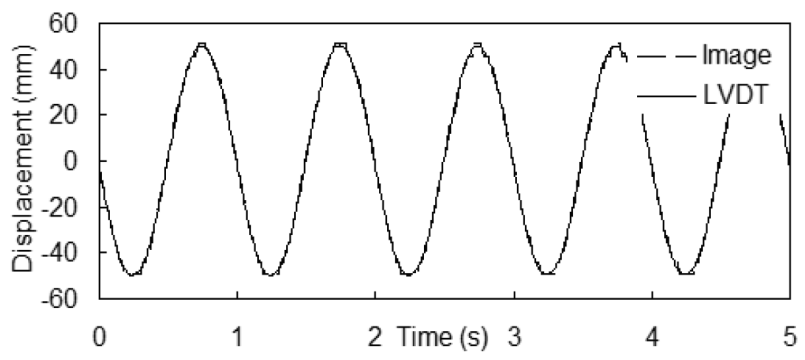

(a)

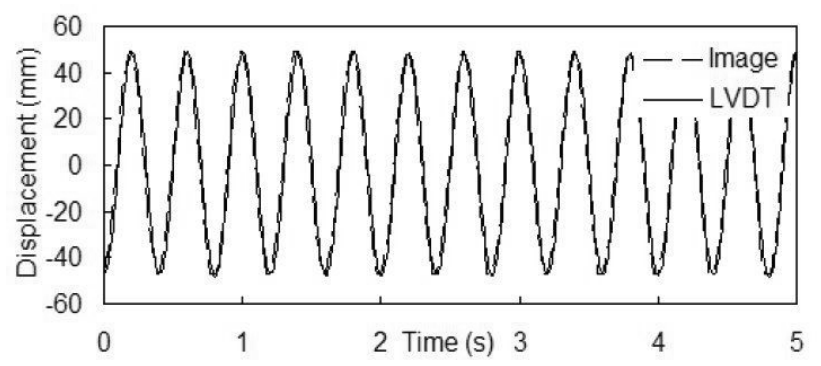

(b)

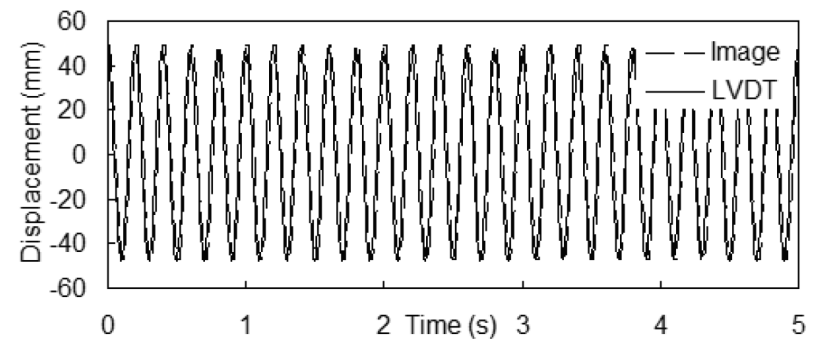

(c)

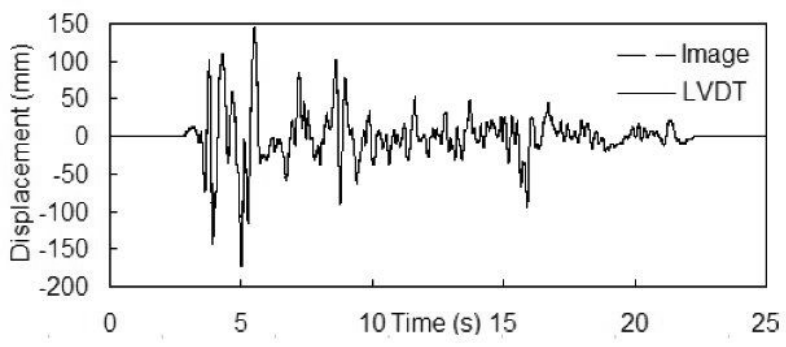

(d)

Fig. 5. Comparison between measured dynamic displacements. (a) $1 \mathrm{~Hz}$ sinusoidal signal. (b) $2.5 \mathrm{~Hz}$ sinusoidal signal. (c) $5 \mathrm{~Hz}$ sinusoidal signal. (d) Earthquake ground motion (Great Hanshin-Awaji earthquake, 1995).

confirmed that with a sub-pixel analysis, further improvement can be expected.

\section{Sub-Pixel Analysis}

In an actual field operation, it is difficult to eliminate the errors, which are caused by a refractive index of air and effect of atmospheric motions during image capturing. In order to reduce such errors, it is important to capture a large area and use a large template image to perform template matching. This can be achieved by using a telescopic lens with a short focal length and preparing an image capture system with a high resolution and wide color depth. However, the total system cost 


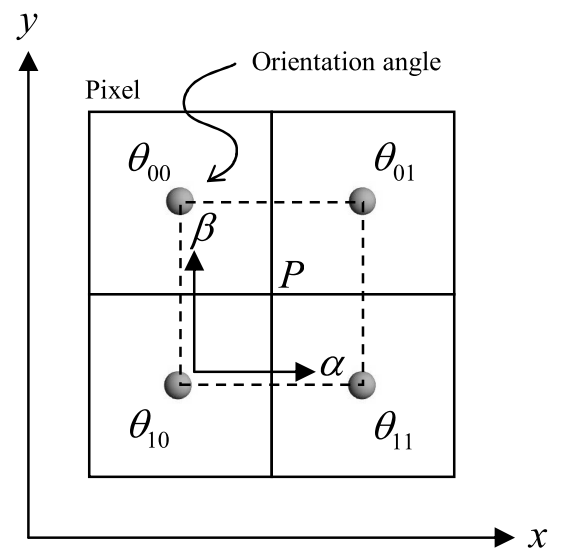

Fig. 6. Sub-pixel analysis.

tends to be expensive by employing these high performance devices.

In this study, in order to cost-effectively reduce measurement errors, a sub-pixel OCM technique was developed. In this method, the OCM algorithm is performed with a sub-pixel resolution accuracy by interpolating obtained orientation angle with a bilinear interpolation. As illustrated in Figure 6, the interpolated orientation angle $\theta$ is obtained as follows:

$$
\begin{aligned}
\theta= & \alpha\left(\beta \theta_{11}+(1-\beta) \theta_{10}\right) \\
& +(1-\alpha)\left(\beta \theta_{01}+(1-\beta) \theta_{00}\right)
\end{aligned}
$$

where $\theta_{00}, \theta_{01}, \theta_{10}$, and $\theta_{11}$ are the orientation angles those surround the grid point $P$. The relative coordinate $(\alpha, \beta)$ represents the position in a sub-pixel resolution. The range of each axis is $[0,1]$. Figure 7 shows the comparison of the time histories measured by the vision-based system with and without the sub-pixel analysis and the time histories measured by a LVDT at $0.1 \mathrm{~Hz}, 2.5 \mathrm{~Hz}$, and $5 \mathrm{~Hz}$ frequency respectively in the shaking table tests. The minimum resolution of the sub-pixel analysis in this experiment is $1 / 10$ pixel. As shown in this figure, in comparison the time history measured by without the sub-pixel analysis and the LVDT, the quantization error is observed clearly. The actual size of the pixel in the image was $0.54 \mathrm{~mm}$ as calibrated in the shaking table test, thus the expected maximum error is $0.27 \mathrm{~mm}$. As this theoretical maximum error, obtained maximum standard deviation was $0.21 \mathrm{~mm}$ and its average of all the tests in different frequency was $0.14 \mathrm{~mm}$. On the other hand, in the results processed with the sub-pixel analysis, the displacement waveforms agree better with the results measured by LVDT. As shown in Figure 8, the average of standard deviation was obtained under $0.05 \mathrm{~mm}$. Moreover, in the actual earthquake shaking test, the standard deviation decreases from $0.14 \mathrm{~mm}$ to $0.043 \mathrm{~mm}$ after employing the sub-pixel analysis. The effectiveness of the sub-pixel OCM technique was confirmed.

\section{Field Test at a LONG-Span BRIDGe}

The performance of the vision-based sensor system in tracking an existing feature on a structure without using a target panel was evaluated through a field experiment at the Vincent Thomas Bridge, a 1500-ft long suspension bridge in Long Beach, CA. As shown in Figure 9, two synchronized

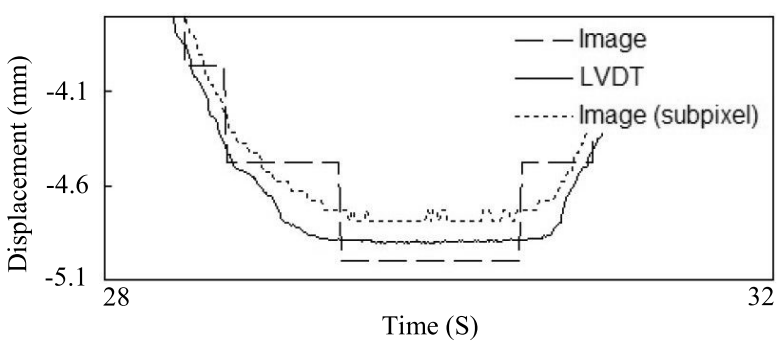

(a)

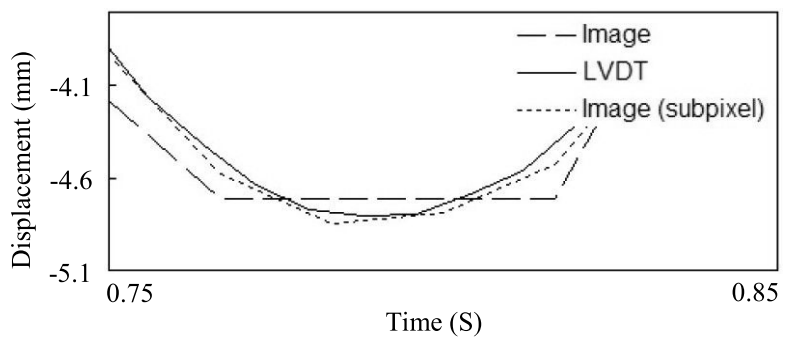

(b)

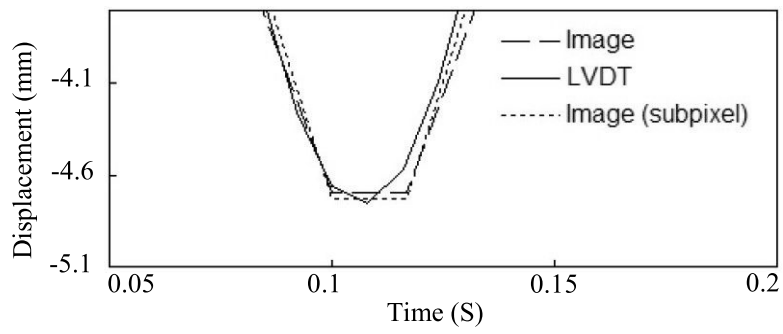

(c)

Fig. 7. Comparison of LVDT, vision-based system w, w/o sub-pixel resolution. (a) $0.1 \mathrm{~Hz}$ sinusoidal signal. (b) $2.5 \mathrm{~Hz}$ sinusoidal signal. (c) $5 \mathrm{~Hz}$ sinusoidal signal.

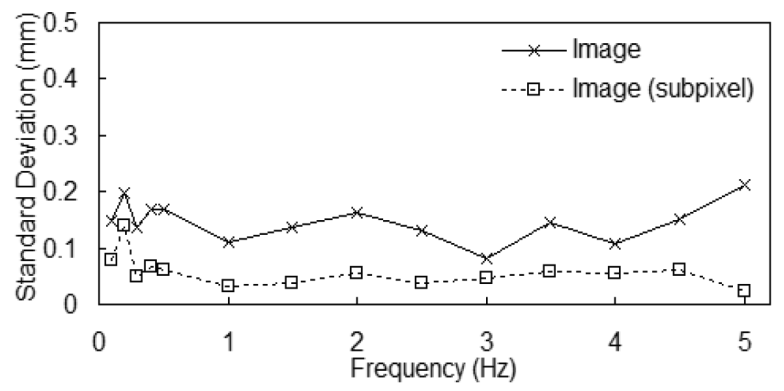

Fig. 8. Reduction of measurement errors.

video cameras were placed at a stationary location approximately $300 \mathrm{~m}$ away from the target at the mid span of the bridge main span. One camera focused on the target panel $(800 \mathrm{~mm} \times 750 \mathrm{~mm})$ with random black and white patterns, which was installed at the mid span, whereas the other targeted existing rivets and edges on the bridge near the target panel.

The vertical displacements of the mid span were measured simultaneously with and without the target panel based on video images from the two cameras. Figure 10 shows the actual images captured by the video camera which focused on the installed target panel and existing rivet pattern close to the target panel respectively. The resolution of the captured 


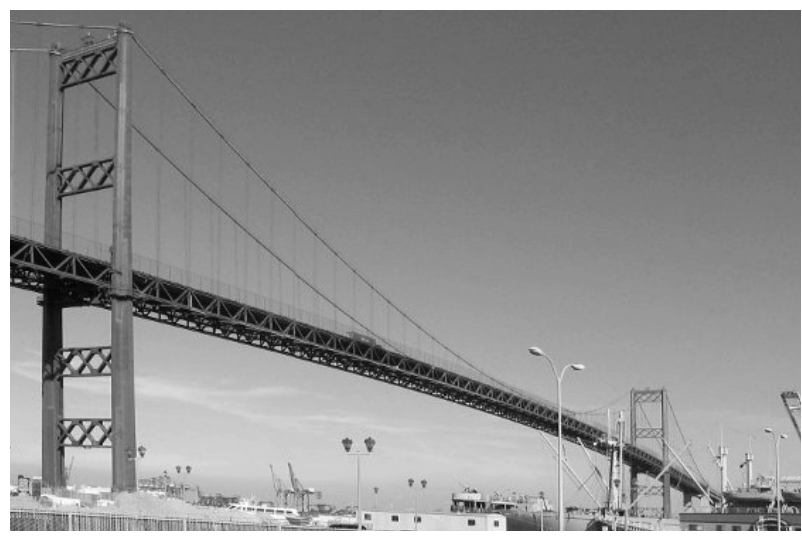

(a)

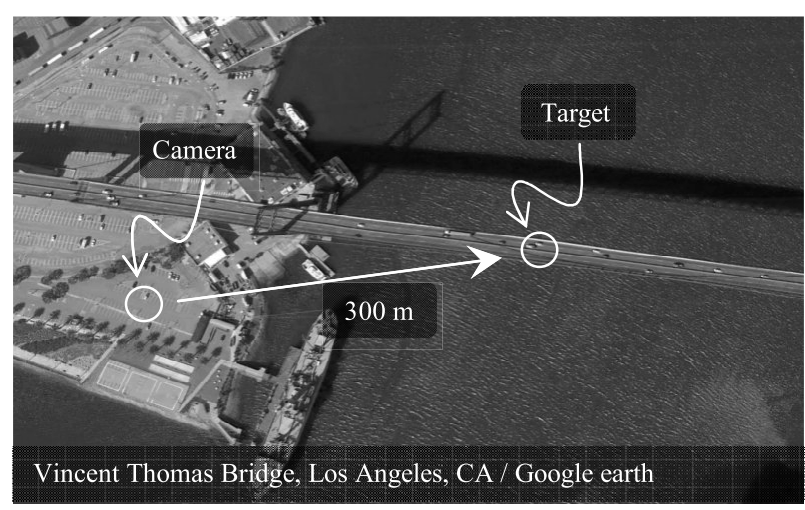

(b)

Fig. 9. Field test at long-span bridge. (a) Vincent Thomas Bridge (Los Angeles, CA). (b) Satellite image of field test and position relation of visionbased system and target position to be measured.

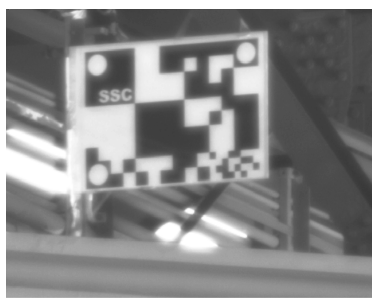
on target panel
Captured by camera focusing

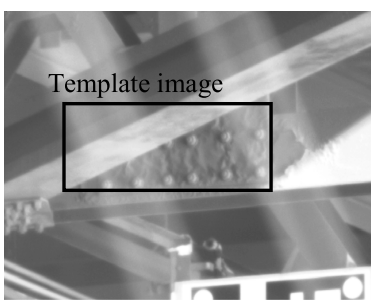

Captured by camera focusing on bolt pattern on the bridge
Fig. 10. Actual image captured by two cameras.

images is $640 \times 480$ pixel and its frame-rate is 60 frames per second. In this field test, the vibration in vertical direction of the bridge is measured. To measure actual length of the amplitude of the vibration, the calibration to obtain the actual pixel size is performed before the measurement by using the target panel, and its size is obtained as $3.01 \mathrm{~mm}$.

Moreover, additional two tests, with different lighting condition, were performed. One is performed in the morning with enough sunlight and the other is performed in the early evening without sufficient illumination to ensure the robustness of the OCM algorithm against of the variation of the light condition. The contrast of these two images was measured in Michelson contrast. In the morning, the contrast was 0.63 and in the evening, 0.36 .

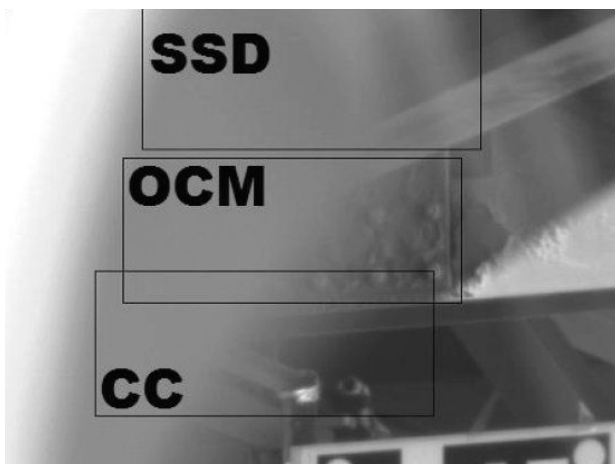

Fig. 11. Comparison of OCM with CC \& SSD in field test.

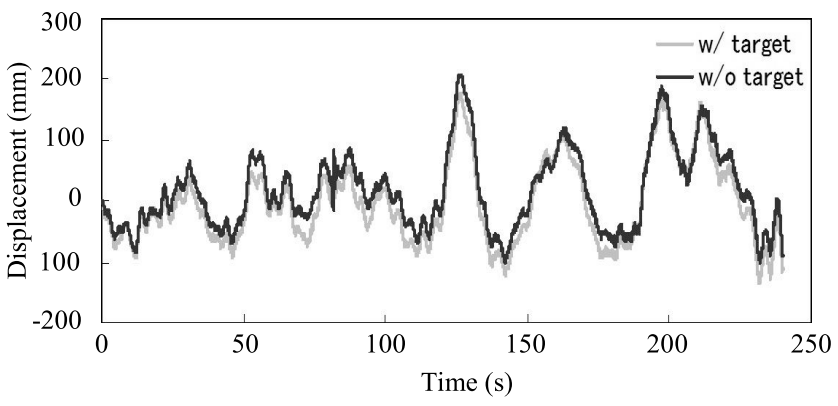

(a)

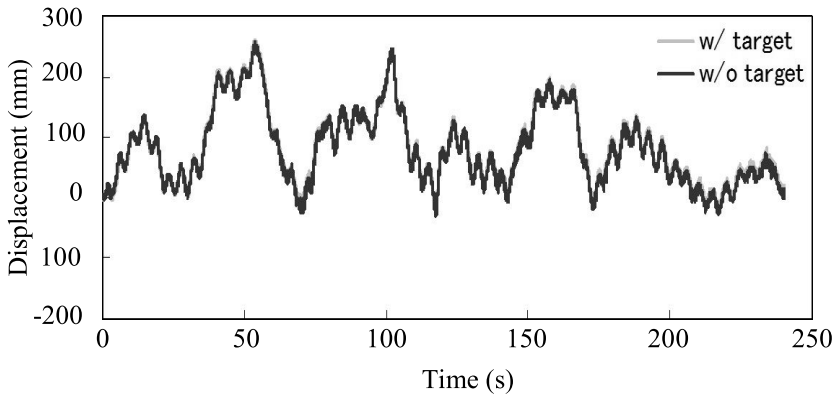

(b)

Fig. 12. Displacement time histories. (a) Displacement time history in the moring. (b) Displacement time history in the evening.

To evaluate the robustness of the OCM algorithm against the environmental noises, which appears in the captured images, an additional experiment was performed, as post-processing, by applying different template matching algorithms including the OCM and the conventional SSD and CC to the measured video images during the field experiment. Figure 11 shows a single frame of the captured image selected for the evaluation of these three different algorithms. This is an image of the same area as in Figure 10, but is partially occluded by tree leaves in the wind. Also the area, where is registered as the template image, is shadowed and the contrast in the area is not high and constant. The detected areas after applying above template matching techniques are represented as black rectangles in Figure 11. As a result, only the matching test with the OCM technique could detect the correct position in the target image successfully. The efficiency of the OCM technique is confirmed also in actual location where various noises could be appeared. 


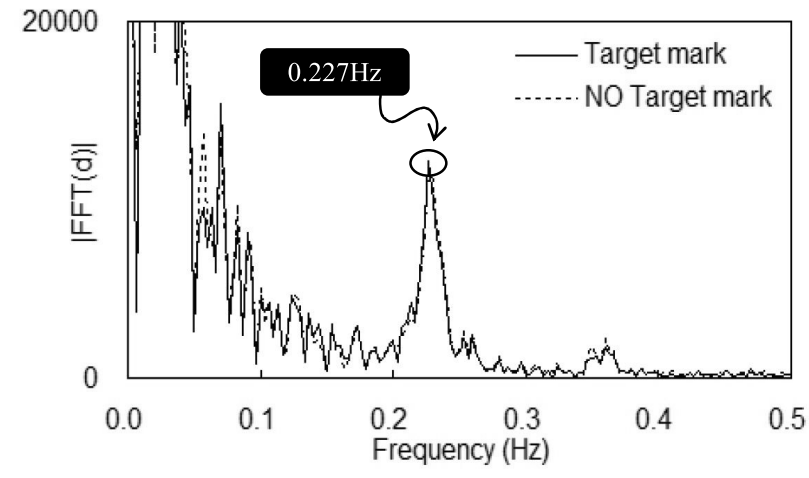

(a)

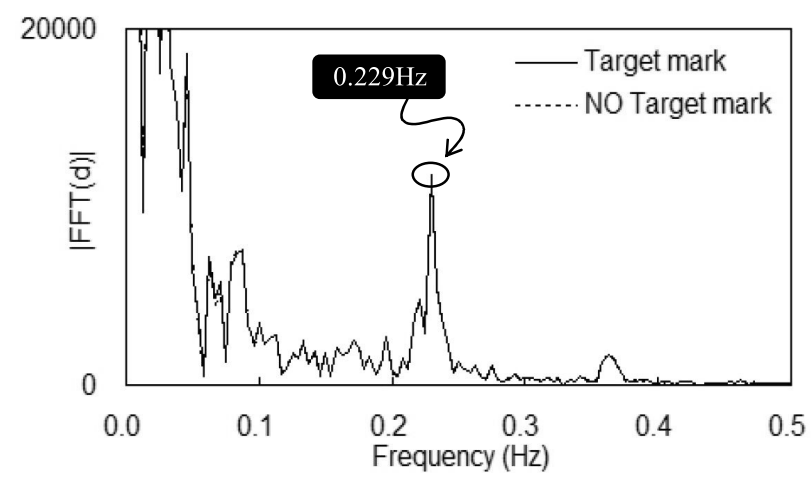

(b)

Fig. 13. FFT amplitudes of time histories. (a) FFT amplitude in the morning. (b) FFT amplitude in the evening.

Figure 12 compares the displacements with and without using the target panel, obtained respectively from the measurement made in the morning and in the evening when there is no sufficient light. The average of the standard deviation between the measured displacements with and without the target panel was $6 \mathrm{~mm}$. The excellent agreement between the results with and without the target panel demonstrates the effectiveness of the proposed OCM algorithm in tracking existing features.

Furthermore, Fourier transform was performed on the measured displacement time histories and the Fourier amplitudes are plotted in Figure 13. Again, the displacements measured with and without using the target panels agreed well in the frequency domain. A dominant frequency, $0.227 \mathrm{~Hz}$ in the morning and $0.229 \mathrm{~Hz}$ in the evening, is observed. There was no difference between the results with and without the target panel. This frequency is consistent with the bridge fundamental frequency measured by accelerometers installed on the bridge [24].

\section{CONCLUSION}

A video camera-based sensor system has been developed for remote measurement of dynamic displacements of large-size structures. By implementing the advanced, robust OCM image processing algorithm, the displacement can be measured with a high-precision by tracking existing features on the structure without requiring the installation of a target mark panel. This represents significant advance over the state of the art, enabling remote monitoring of structures with difficult access, as well as the flexibility of changing measurement points. The shaking table tests involving sinusoidal and seismic motions demonstrate the accuracy of the dynamic displacement measurement through comparison with an LVDT, a conventional contact sensor. The measurement errors are further reduced by a sub-pixel OCM technique proposed in this study. The high performance of the sensor system is further demonstrated through a test on a long-span suspension bridge in the field, in which the bridge displacement time histories measured 300 meters away by tracking existing features on the bridge achieve an excellent agreement with those by tracking a target panel. The field test also shows the robustness of the OCM image processing to insufficient illumination conditions in the field. Future work will be carried out to investigate the integration of the OCM with other template matching-based image processing techniques, such as the DIC technique to further improve the displacement measurement performance. The availability of such a high-performance remote sensor system will enable cost-effective displacement measurement of large-size structures in the field under operational loads, which can be used for monitoring their structural integrity.

\section{ACKNOWLEDGMENT}

The authors would like to thank the California Department of Transportation for providing the Vincent Thomas Bridge and technical support to the field tests.

\section{REFERENCES}

[1] B. C. Faulkner, W. B. Furman, T. B. Thomas, and T. M. Wallace, "Determination of bridge response using acceleration data," Virginia Dept. Transp., Richmond, VA, USA, Tech. Rep. FHWA/VA-97-R5, 1996.

[2] K. T. Park, S. H. Kim, H. S. Park, and K. W. Lee, "The determination of bridge displacement using measured acceleration," Eng. Struct., vol. 27, no. 3, pp. 371-378, 2005.

[3] G. W. Roberts and A. H. Dodson, "A remote bridge health monitoring system using computational simulation and GPS sensor data," Seismol. Soc. Amer, vol. 25, no. 4, pp. 299-308, 1998.

[4] M. Çelibi, "GPS in dynamic monitoring of long-period structures," Soil Dyn. Earthquake Eng., vol. 20, no. 5, pp. 477-483, 2000.

[5] S. Nakamura, "GPS measurement of wind-induced suspension bridge girder displacements," J. Struct. Eng., vol. 126, no. 12, pp. 413-1419, 2000.

[6] A. Knecht and L. Manetti, "Using GPS in structural health monitoring," Proc. SPIE, vol. 4328, p. 122, Aug. 2001.

[7] L. Xu, J. J. Guo, and J. J. Jiang, "Time-frequency analysis of a suspension bridge based on GPS," J. Sound Vibrat., vol. 254, no. 1, pp. 105-116, 2002.

[8] H. H. Nassif, M. Gindy, and J. Davis, "Comparison of laser doppler vibrometer with contact sensors for monitoring bridge deflection and vibration," NDT \& E Int., vol. 38, no. 3, pp. 213-218, 2005.

[9] E. S. Bell, J. T. Peddle, and A. Goudreau, "Bridge condition assessment using digital image correlation and structural modeling," in Proc. 6th Int. Conf. Bridge Maintenance, Safety Manag., 2012, pp. 330-337.

[10] C. Warren, P. Pingle, C. Niezrecki, and P. Avitable, "Comparison of image based, laser, and accelerometer measurements," in Proc. Conf. Soc. Exp. Mech. Ser, vol. 12. 2011, pp. 15-21.

[11] Y. F. Ji and Q. W. Zhang, "A novel image-based approach for structural displacement measurement," in Proc. 6th Int. Conf. Bridge Maintenance, Safety Manag., 2012, pp. 407-414.

[12] Y. Fukuda, M. Feng, and M. Shinozuka, "Cost-effective vision-based system for monitoring dynamic response of civil engineering structures," Struct. Control Health Monitor, vol. 17, no. 8, pp. 918-936, 2009.

[13] E. Caetano, S. Silva, and J. Bateira, "A vision system for vibration monitoring of civil engineering structures," Exp. Tech., vol. 35, no. 4, pp. 74-82, Jul./Aug. 2011. 
[14] S. W. Kim, N. S. Kim, and Y.-M. Kim, "Application of vision-based monitoring system to stay cables," in Proc. 6th Int. Conf. Bridge Maintenance, Safety Manag., 2012, pp. 1116-1123.

[15] M. A. Iadicola, R. S. Zobel, and J. M. Ocel, "Quantitative evaluation of digital image correlation as applied to large scale gusset plate experiments," in Proc. 6th Int. Conf. Bridge Maintenance, Safety Manag., 2012, pp. 1436-1443.

[16] G. Busca, A. Cigada, P. Mazzoleni, E. Zappa, and M. Franzi, "Cameras as displacement sensors to get the dynamic motion of a bridge: Performance evaluation against traditional approaches," in Proc. 6th Int. Conf. Bridge Maintenance, Safety Manag., 2012, pp. 2835-2841.

[17] M. M. Gorkani and R. W. Picard, "Texture orientation for sorting photos at a glance," ACM Comput. Surveys, vol. 24, no. 4, pp. 325-376, 1992.

[18] W. Freeman and M. Roth, "Orentation histograms for hand gesture recognition," in Proc. IEEE Int. Workshop Face Gesture Recog., Jun. 1995, pp. 296-301.

[19] S. Kaneko, I. Murase, and S. Igarashi, "Robust image registration by increment sign correlation," Pattern Recog., vol. 35, no. 10, pp. 2223-2234, 2002.

[20] F. Ullah and S. Kaneko, "Using orientation codes for rotation-invariant template matching," Pattern Recognit., vol. 37, no. 2, pp. 201-209, 2004.

[21] F. Ullah, S. Kaneko, and S. Igarashi, "Orientation code matching for robust object search," IEICE Trans. Inf. Syst., vol. E84-D, no. 8, pp. 999-1006, Aug. 2001.

[22] Y. Li, H. Takauji, S. Kaneko, T. Tanaka, and I. Ohmura, "Robust focusing using orientation code matching," Electron. Lett. Comput. Vis. Image Anal., vol. 7, no. 3, pp. 101-114, 2008.

[23] Y. Narita, "Remote and robust displacement measurement based on picture matching," M.S. thesis, Hokkaido Univ., Sapporo, Japan, 2010.

[24] M. Shinozuka, D. Karmakar, S. R. Chaudhuri, and H. Lee, "Verification of computer analysis models for suspension bridges," California Dept. Transportation, Tech. Rep. CA/UCI-VTB-2009, 2009.

Yoshio Fukuda was born in Yamaguchi, Japan, in 1976. He received the B.S. degree in product system engineering from the Ube National College of Technology, Yamaguchi, in 1999, and the M.S. and Ph.D. degrees in mechanical engineering from Nagasaki University, Nagasaki, Japan, in 2001 and 2004, respectively.

He is currently an Associate Research Scientist with the Civil Engineering and Engineering Mechanics Department, Columbia University, Columbia, NY, USA. His current research interests include visual sensing, man machine interface, human body sensing devices, and civil structure health monitoring.
Maria Q. Feng received the Ph.D. degree in mechanical engineering from the University of Tokyo, Tokyo, Japan, in 1992. She is an expert in safety and sustainability of civil infrastructure systems and military armor protective systems, with an emphasis on innovative and interdisciplinary science and technology for sensors, structural health monitoring, and damage assessment. She has pioneered the development of novel fiber optic and vision-based sensors, microwave imaging technology, wind/earthquake/blast protective systems, as well as vibration-based system identification algorithms for health diagnostics of buildings and bridges. She directs the Sensing, Monitoring, and Robotics Technology Laboratory. She is a fellow of the American Society of Civil Engineers (ASCE). She has received numerous national and international awards in recognition of her research achievements, including the National Science Foundation CAREER Award, the ASCE's Collingwood Prize and Walter L. Huber Civil Engineering Research Prize, and the Alfred Noble Prize, which is given jointly by the ASCE and other professional societies.

Yuto Narita was born in Hokkaido, Japan, in 1987. He received the B.S. degree in computer science from Hokkaido University, Sapporo, Japan, in 2010. He is currently a Machine Designer with the Machine Development Division, Toyota Motor Corporation, Aichi, Japan. His current research interests include the development of machines for the production of new electronic devices.

Shun'ichi Kaneko received the B.S. degree in precision engineering and the M.S. degree in computer science from Hokkaido University, Sapporo, Japan, and the Dr.Eng. degree from Tokyo University, Tokyo, Japan, in 1978 and 1980, respectively. He joined the Computer Science Department, TUAT in 1980 and has been a Professor with Hokkaido University since 2004. His current research interests include pictorial pattern recognition, robust statistical information processing, and visual sensing. He received the Best Paper Award, the Society Award of JPSE, and the Odawara Memorial Award for Best Paper in VIEW in 2000.

Takayuki Tanaka received the B.S., M.S., and Ph.D. degrees in mechanical engineering from the University of Electro-Communications (UEC), Tokyo, Japan, in 1994, 1996, and 1999, respectively. He joined the Intelligent Mechanical Department, UEC, in 1996 and has been an Associate Professor with Hokkaido University, Sapporo, Japan, since 2004. His current research interests include wearable robot, power assist system, and image measurement. He received the Best Paper Award of SICE and the Human Interface Society. 